\title{
Quantifying Behavioral Changes in Territorial Animals Caused by Sudden Population Declines
}

\author{
Jonathan R. Potts, ${ }^{1,2}$ Stephen Harris, ${ }^{2, *}$ and Luca Giuggioli ${ }^{1,2,3}$ \\ 1. Bristol Centre for Complexity Sciences, University of Bristol, Bristol, United Kingdom; 2. School of Biological Sciences, University of \\ Bristol, Bristol, United Kingdom; 3. Department of Engineering Mathematics, University of Bristol, Bristol, United Kingdom
}

Submitted January 29, 2013; Accepted March 19, 2013; Electronically published July 12, 2013

Online enhancements: appendix, videos. Dryad data: http://dx.doi.org/10.5061/dryad.5dn48

AвSTRACT: Although territorial animals are able to maintain exclusive use of certain regions of space, movement data from neighboring individuals often suggest overlapping home ranges. To explain and unify these two aspects of animal space use, we use recently developed mechanistic models of collective animal movement. We apply our approach to a natural experiment on an urban red fox (Vulpes vulpes) population that underwent a rapid decline in population density due to a sarcoptic mange epizooty. By extracting details of movement and interaction strategies from location data, we show how foxes alter their behavior, taking advantage of sudden population-level changes by acquiring areas vacated due to neighbor mortality, while ensuring territory boundaries remain contiguous. The rate of territory border movement increased eightfold as the population declined and the foxes' response time to neighboring scent reduced by a third. By demonstrating how observed, fluctuating territorial patterns emerge from movements and interactions of individual animals, our results give the first data-validated, mechanistic explanation of the elastic disc hypothesis, proposed nearly 80 years ago.

Keywords: animal movement, home range, red fox (Vulpes vulpes), epizooty, territoriality, theoretical ecology.

\section{Introduction}

Much has been written about the factors affecting territory size, such as allometry, resource dispersion and availability, and population density (Kruuk and Parish 1982; Grant and Kramer 1990; Jetz et al. 2004; Moorcroft and Barnett 2008; Van Moorter et al. 2009; Schradin et al. 2010). Despite this, we know remarkably little about how territories form and change shape (Adams 2001) and how animals interact to maintain these territories (Börger et al. 2008). Providing this understanding is of great importance to many areas of ecology, from conservation biology to wildlife management and from predator-prey dynamics to ep-

\footnotetext{
* Corresponding author; e-mail: s.harris@bristol.ac.uk.
}

Am. Nat. 2013. Vol. 182, pp. E73-E82. (c) 2013 by The University of Chicago. 0003-0147/2013/18203-54436\$15.00. All rights reserved. DOI: $10.1086 / 671260$ idemiology (Beier 1993; Lewis Murray 1993; Kenkre et al. 2007; McCarthy and Destefano 2011). Though such applications often assume that territories are roughly stationary, population density can change rapidly in a variety of situations, such as when a population is suffering an epizooty of a terminal disease, thereby affecting the territorial structure and behavior of the individual animals. During 1994-1996, a sarcoptic mange epizooty decimated the red fox (Vulpes vulpes) population in Bristol, causing changes in both the movement of the foxes and territory sizes (Baker et al. 2000). This provided support to the idea that territories deform elastically, which was first observed nearly 80 years ago by Huxley (1934) in coot (Fulica atra) populations. While elastic territories have since been detected in a variety of species, such as martins (Progne subis; Stutchbury 1991), warblers (Acrocephalus arundinaceus; Ezaki 1995), and lizards (Anolis aeneus; Stamps and Krishnan 1998), construction of a mechanistic theory that underpins this elasticity has tended to remain elusive.

In the context of scent-marking animals, the process with which animals respond to the information present in scent deposited by a conspecific is key to the correct quantification of territorial dynamics. From the movement perspective of the individual animal, this is a binary choice between ignoring foreign scent, if the information contained in it is either old or uninformative, or retreating. A given location thus either has or does not have an "active scent," that is, a scent that is responded to by conspecifics as a fresh territory cue. The presence/absence nature of the scent implies that the system is intrinsically stochastic, so deterministic representation via reaction-diffusion formalisms may be unable to account for the discrete nature of the interaction events (e.g., see Durrett and Levin 1994; McKane and Newman 2004). A recent modeling framework (Giuggioli et al. 2011a, 2011b, 2012; Potts et al. 2011, 2012) that accounts for the discrete nature of the scentmediated interaction events is employed here to interpret 
observations of the Bristol fox population before and during the 1994-1996 mange epizootic.

Some territorial animals have a strong drift tendency toward the locations of their den sites and Moorcroft et al. (2006) examined how these territorial patterns change when a coyote (Canis latrans) pack is removed from a population. Making use of a reaction-diffusion formalism where the position densities of two neighboring packs are coupled with density profiles of the scent marks and with a predetermined knowledge of the locations of the coyote den sites, Moorcroft and Lewis (2006) compared the steady state position distributions of the animals before and after the removal. This approach, however, cannot be used in our context because the tendency to drift toward the den site is either not present in Bristol's foxes or not sufficiently strong to generate steady state position distributions, since the mean square displacement of the animals increases with time and never settles (Giuggioli et al. 2011a). Neither was the habitat spatially confined, as in Briscoe et al. (2002). One of the fundamental advancements of the stochastic framework proposed by Giuggioli et al. (2011a) is the ability to quantify the (discrete) longevity of scent cues and the movements in territory borders, a key notion implicit in the elastic disc hypothesis. See Potts et al. (2012) for a detailed comparison of the two approaches.

By using the more recent approach, we quantify elasticity in territorial patterns by the use of a single parameter $K$, the diffusion constant of the territory border, measuring the rate at which the variance of border positions increases over time. Variations in mean territory size, the amount of directional persistence in the animal movement process, and the animal velocity are also quantified, enabling behavioral changes due to a sudden population decline to be assessed. Additionally, we analyze agent-based simulations of systems of moving and interacting animals (Giuggioli et al. 2011a), to determine the longevity of territorial scent marks, the active scent time $T_{\mathrm{AS}}$, from the information provided in the parameter $K$. Animals are modeled to move at random (Okubo and Levin 2002) but constrained to roam within areas that do not contain scent of conspecifics. As each animal moves, it deposits scent, but once the scent has been present for time $T_{\mathrm{AS}}$, it is no longer considered by others to be fresh and so is ignored. In the field, while scent marks cannot persist after the chemicals have decayed or dispersed, it may be beneficial for animals to intrude into a neighboring territory if the odor of the scent mark they detect is old, suggesting that the territory may no longer be defended. To test whether this happens in the Bristol fox population, we showed that the active scent time decreased after the outbreak of mange, demonstrating that $T_{\mathrm{AS}}$ must arise from a behavioral strategy rather than being solely a consequence of the persistence of the chemicals in the environment. This decrease might also be influenced by a ces- sation of vocal cues from recently dead neighbors (Newton-Fisher et al. 1993).

\section{Methods}

\section{Stochastic Simulations}

Stochastic simulations were performed based on the twodimensional (2-D) territorial random walk model of Giuggioli et al. (2011a) but employing one of two different movement processes: nearest-neighbor random walks (NNRWs) and ballistic walks (BWs). The NNRW process is described in Giuggioli et al. (2011a), whereas ballistically moving animals will always continue in a straight line, unless they encounter foreign scent, causing them to turn at random. For each simulation run, 25 animals were placed on a 2-D square grid size $M \times M$ with lattice spacing $a$ and population density $\rho=25 / M^{2}$, so that the grid is approximately 25 times the size of a territory. While the dominant pair share the same territory (Saunders et al. 1993) and territory configuration could be calculated from any individual within a group (Baker et al. 2000), the simulations only modeled one animal per territory, the dominant male, so when fitted to the data, $\rho$ was the population density of dominant males.

All simulated animals moved with the same movement process, NNRW or BW, constrained by the fact that each animal could not enter a square that contained fresh scent of a different animal, and so turned away from the square in a random direction. Each animal deposited scent at every square it visited, which remained for a finite time $T_{\mathrm{AS}}$, the active scent time. After this time period had elapsed, the scent was no longer recognized by conspecifics as a fresh scent message and so was no longer present in the simulation. The squares that contained active scent of an animal constituted its territory and the locations where two contiguous territories met made a territory border. The speed of each animal was $v$, and $\tau=a / v$ was the time it took for an animal to move distance $a$.

While certain animals are so-called borderland markers, for example, badgers (Meles meles; Hutchings et al. 2001), who actively patrol their borders to discourage invaders, foxes are hinterland markers (Macdonald 1980), meaning they deposit scent marks evenly throughout their territory. Our previous studies have shown that greater patrolling of the borders can reduce the amount they shift (Giuggioli et al. 2011a), and we conjectured that foxes might be adopting such a strategy. However, closer analysis of the relative amount of time foxes spend near their territory borders reveals that this is not the case (see "Methods for Inferring Time Spent at the Territory Border," available online). Therefore, we have not included active border patrolling in this model. 


\section{A Reduced Analytic Approximation of the Simulation Model}

To fit data to the model efficiently, we used an analytic approximation from Giuggioli et al. (2012) that models the movement of a correlated random walker (CRW; animal) inside a moving square territory of average width $L$, which is equal to $1 /(\rho)^{1 / 2}$ (see fig. $1 a$ for a pictorial explanation). To measure the amount of time an animal has directional persistence, we used the correlation time $\mathcal{T}$ (Viswanathan et al. 2005). This has the following practical interpretation. If position fixes from a CRW with correlation time $\mathcal{T}$ were taken at time intervals greater than $\mathcal{T}$, analyzing the turning angles between the position fixes would suggest that the animal was moving in an uncorrelated fashion. Conversely, if the fixes were taken at time intervals less than $\mathcal{T}$, turning angle analysis would suggest that the movement was correlated.

As territories exclude one another, the time dependence of the territory border mean square displacement (MSD), that is, the variance of the occupation probability, is slightly sublinear. This occurs due to an exclusion process, well studied in the statistical physics literature, for example, Liggett (1985), but only recently introduced into the literature on animal territoriality (Giuggioli et al. 2011a). An exclusion process is one where there are multiple moving objects, in our case territories, that cannot either overlap or occupy the same place at the same time. Since the territories hamper each others' movement, the MSD does not grow linearly in time (free diffusion). In fact, Landim (1992) has indicated that 2-D exclusion processes have an asymptotic (i.e. long-time) MSD proportional to $t / \ln (t)$. Therefore, we assume that the output of the territory border movement in the simulation model is asymptotically equal to $2 \mathrm{Kt} / \ln (t / \tau)$. The reason for dividing by the constant $\tau$ here is to ensure $K$ has the units of a diffusion constant (space $2 /$ time), which becomes convenient later when we compare it with the diffusion constant $v^{2} \tau$ of the animal (e.g., see fig. 2). The analytic approximation model has the territory border diffusion constant $K$ as an input parameter.

Since larger-than-average territories in the simulation model tend to shrink but smaller-than-average ones tend to grow, the analytic model contains a rate parameter $\gamma$ that measures the strength of this tendency (fig. 1a). We have summarized the various parameters in the model in table 1 . The data were fitted to the probability density function $P(x, y, t \mid \theta)$ for the animal to be at coordinates $(x, y)$ relative to its home range center, defined as the centroid of all the measured positions of the animal over the study period, at time $t$, given the input parameters $\boldsymbol{\Theta}=(v, K, \mathcal{T}, \gamma, L)$ (see "Analytic Expression of the Probability Distribution for an Animal in a Slowly Moving
Territory," available online for the full analytic expression of $P(x, y, t \mid \theta)$ and its derivation).

\section{Calculating Home Range Overlap from Territory Border Movement}

The amount of overlap between neighboring home ranges was calculated by assuming that the two territories share a common edge. In the model, this edge is continuously moving, creating an overlap in the space used by the two adjacent animals when measured over a time interval $T_{*}$. For example, if an edge between two territories, each modeled as a square of side $L$ (see fig. 1), moves a distance of $\Delta L$ in the perpendicular direction during the time $T_{*}$, then the overall area shared by these animals in two territories during this time is $L \times \Delta L$. In practice, $T_{*}$ represents the time window during which location data are collected. As the territory borders move during this time window, we observe overlaps in the home ranges measured from the locations of animals in neighboring territories (fig. 1b).

In our model, the MSD in the perpendicular direction of the shared edge between two adjacent territories is $K t / \ln (t / \tau)$. Therefore, the width of the overlapping strip between the two neighboring home ranges is equal to the mean absolute displacement, which is $\left[K T_{*} / \ln \left(T_{*} / \tau\right)\right]^{1 / 2}$. The width of the home range is then $1 /(\rho)^{1 / 2}+$ $\left[K T_{*} / \ln \left(T_{*} / \tau\right)\right]^{1 / 2}$, owing to the fact that the width of each territory is $1 /(\rho)^{1 / 2}$. Therefore, the fraction of a home range that overlaps with this particular neighbor is

$$
\mathrm{HRO}=1-\frac{1}{1+\sqrt{K T_{*} \rho / \ln \left(T_{*} / \tau\right)}} .
$$

Notice that as $T_{*}$ increases, the fraction of overlap increases toward the theoretical maximum value of 1 , where the two home ranges coincide. However, since territory border movement is typically very slow, the time it would take to get close to this situation is likely to be far longer than the lifetime of the animal. Therefore, complete overlap is highly unlikely to be observed in reality.

\section{Data Collection and Analysis}

Movement data were taken from a long-term study of the red fox population in the Bristol urban area. Our data are available in the Dryad Digital Repository, http://dx.doi .org/10.5061/dryad.5dn48 (Potts et al. 2013). Radio fixes with a spatial resolution of $25 \mathrm{~m} \times 25 \mathrm{~m}$ were taken every 5 min between 20:00 and 04:00 GMT, which encompasses most fox activity (Saunders et al. 1993), so throughout this article " 1 day" is equal to $8 \mathrm{~h}$ of fox location data. Radio telemetry data from 22 different territorial adult foxes (i.e., >1 year old) monitored between 1990 and 1995 
(a)

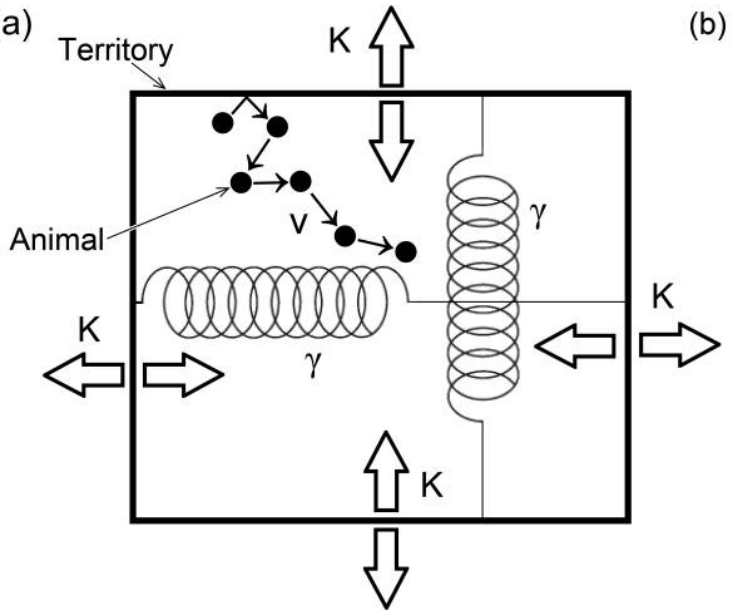

(b)

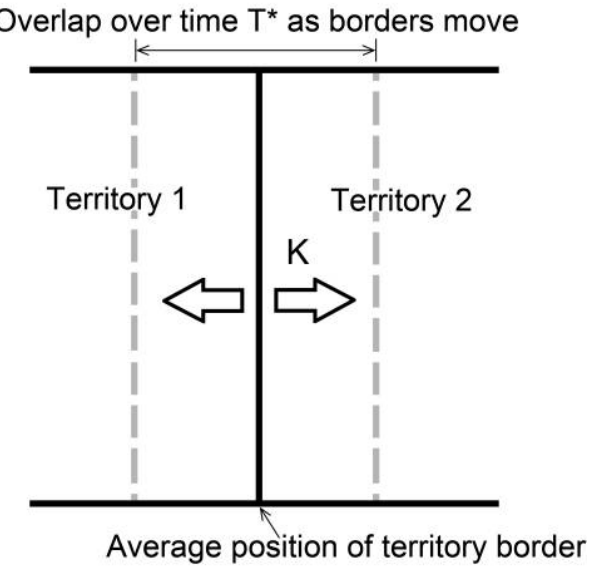

Figure 1: Representation of the approximate analytic model of animal movement within a dynamic territory. Territory borders move randomly with a mean square displacement of $2 K t / \ln (t / \tau)$ around an average width of $L=1 /(\rho)^{1 / 2}$, where $\rho$ is the population density. The process that keeps the territories at this average width is represented by two springs, one vertical and one horizontal, each having a spring constant of $\gamma$. The higher $\gamma$, the greater the tendency for larger- or smaller-than-average territories to move back toward an average size. The animal, represented by a filled circle in $a$, moves as a correlated random walker with speed $v$ and correlation time $\mathcal{T}$ (see "Methods"). Panel $a$ represents this setup, while $b$ demonstrates how overlaps between adjacent home ranges arise from this model as animal positions are measured over time. In $b$, the mean position of the territory border is represented by the solid black line, while the average extent of the movement of this border to the left and right is represented by the dashed gray lines.

were analyzed. Data from both males and females were used because the space use distributions of a male and a female from the same group are very similar (Baker et al. 2000). Each fox was tracked during one, two, or three seasons (spring, March-May; summer, June-August; autumn, September-November; winter, December-February; see table Al, available online). Starting in the summer of 1994, a mange epizootic spread through Bristol's foxes causing the population density to decline rapidly, eventually killing almost all the foxes in the city (Baker et al. 2000). When analyzing the data, we split them into two sets: premange, before summer 1994 when the population density was relatively stable, and postmange, after summer 1994, when the population was rapidly declining. The premange data set contained $N=8,693$ data points from 18 different foxes, postmange $N=2,313$ from 4 foxes (see table A1). The last fox in the study area died in spring 1996 (Baker et al. 2000).

The log maximum likelihood method was employed to fit data to the theoretical probability distribution $P(x, y, t \mid \Theta)$. In particular, the Nelder-Mead simplex algorithm (Lagarias et al. 1998) was used to find the maximum of $\mathcal{L}(\Theta)=\sum_{n} \ln \left[P\left(x_{n}, y_{n}, t_{n} \mid \Theta\right)\right]$ for each set of parameter values $\theta$, where the sum is taken over $99 \%$ of position-time locations $\left(x_{n}, y_{n}, t_{n}\right)$ that attain the highest $P\left(x_{n}, y_{n}, t_{n} \mid \Theta\right)$ values. We excluded $1 \%$ of outliers to ensure the results were not biased by anomalous behavior (Harris et al. 1990; Kenward et al. 2001). The Nelder-Mead al- gorithm requires a starting value for $\theta$, called $\theta_{0}=$ $\left(v_{0}, K_{0}, \mathcal{T}_{0}, \gamma_{0}, L_{0}\right)$, that is expected to be close to the maximum. We set $v_{0}$ to be the total distance moved by the foxes divided by the total time moved. Term $\mathcal{T}_{0}$ was obtained by the formula $\mathcal{T}_{0}=-5 / \ln [\langle\cos (\theta)\rangle] \mathrm{min}$, where $\langle\cos (\theta)\rangle$ is the mean of the cosines of the turning angles $\theta$ from the data (Viswanathan et al. 2005). The factor of 5 comes about since location measurements were taken every $5 \mathrm{~min}$. Since the long-time MSD of the animal is taken to be $2 K t / \ln (t / \mathcal{T})$ (Giuggioli et al. 2012, eq. 3.3), $K_{0}$ was obtained by fitting a curve $A+2 K_{0} t / \ln \left(t / \mathcal{T}_{0}\right)$ to the fox MSD against time $t$ for $t>1$ day, using the least squares method, where $A$ is a fitting constant.

Term $L_{0}$ was found by taking the square root of the mean $100 \%$ minimum convex polygon (MCP) home range area (Harris et al. 1990). While the MCP method is in general not the most accurate for finding home range sizes (Fieberg and Börger 2012), we use it only to find a starting point for running the Nelder-Mead algorithm. Though a more accurate method, for example, kernel density estimation (KDE; Laver and Kelly 2008), may cause the algorithm to converge slightly more quickly, the estimation method we use to obtain the algorithm's initial condition makes no difference to the outcome of the algorithm. Since MCP has the advantage of being very simple to measure from the data and is known to be an accurate measure of home range area and territory boundaries in this particular fox population (Saunders et al. 1993), this is the method 


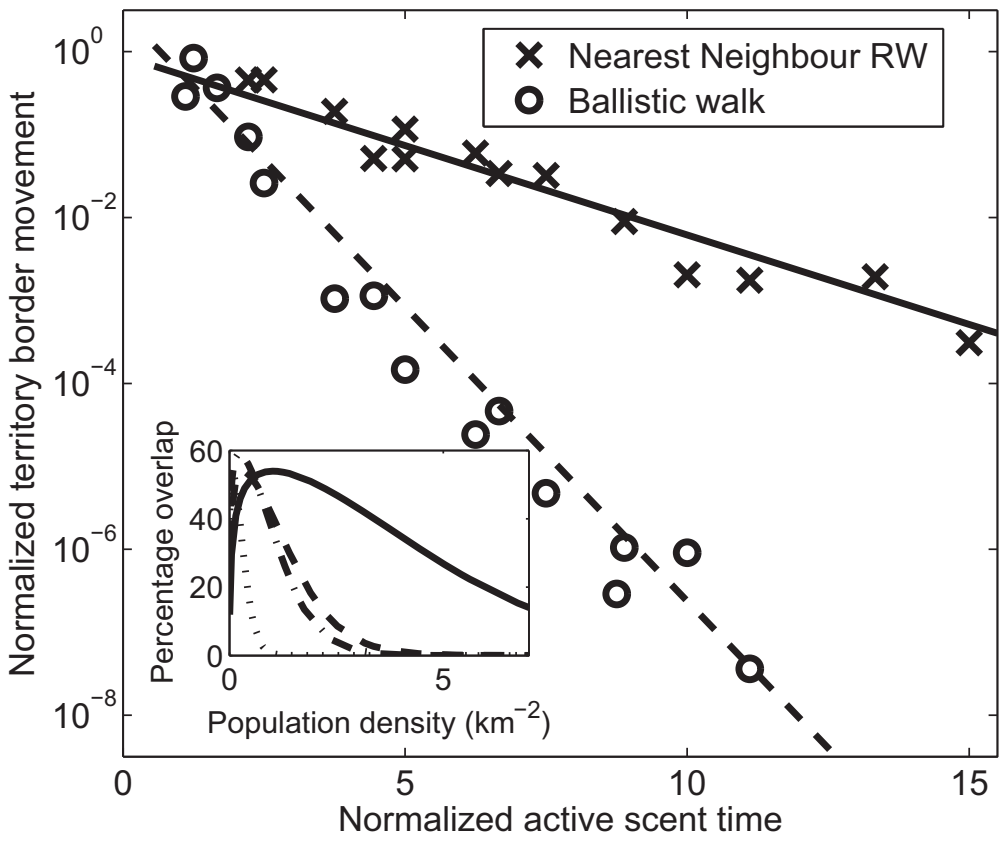

Figure 2: Simulation output showing the dependence of territory boundary movement on the active scent time for nearest-neighbor random walks (NNRWs) and ballistic walks (BWs). The vertical axis is $K / v^{2} \tau$, and the horizontal is $Z=T_{\mathrm{As}} v^{2} \tau \rho$ (see table 1 for definitions of terms). The crosses and circles show values from the simulation output. The solid line is a best fit for the NNRW $\operatorname{case} \log _{10}\left(K / v^{2} \tau\right)=0.085-$ $0.247 Z$, and the dashed line is the best fit for the BW case $\log _{10}\left(K / v^{2} \tau\right)=0.467-0.709 Z$. Inset, the percentage of each home range that overlaps with neighboring ranges. The solid (NNRW) and dashed (BW) lines use fixed values of $T_{\mathrm{AS}}, v, T_{*}$, and $\tau=\mathcal{T}$ taken from the premange data, whereas the dotted (BW) and dot-dashed (NNRW) lines use postmange data.

we choose here. Finally, to find $\gamma_{0}$, the maximum of $\mathcal{L}(\Theta)$ was calculated for $\Theta=\left(v_{0}, K_{0}, \mathcal{T}_{0}, \gamma, L_{0}\right)$ as $\gamma$ varies across parameter space from $\gamma=10^{-3}$ to $\gamma=10^{4}$. Error bars for the best fit were obtained using the bootstrap algorithm for variance calculation (see, e.g., Wasserman 2004) by resampling each data set 100 times.

\section{Results}

The Effect of Movement Processes on Territorial Dynamics

For both of the movement processes simulated, NNRW and $\mathrm{BW}$, the borders of the emergent territories each had an MSD that increased asymptotically as $2 K t / \ln (t / \tau)$. The magnitude of the diffusion constant $K$ depended on the directionality of the animal's movement, the population density $\rho$ and the active scent time $T_{\mathrm{AS}}$ (fig. 2). For each movement process, $K$ depended upon the ratio $Z=$ $T_{\mathrm{AS}} / T_{\mathrm{C}}$ between the active scent time and $T_{\mathrm{C}}=1 /\left(v^{2} \tau \rho\right)$, the territory coverage time, representing how long it takes for an animal to move around its territory (Potts et al. 2012). For the NNRW case, $T_{\mathrm{C}}$ is closely related to the mean time for an animal in a confined region to return to the place from which it started (Condamin et al. 2007).

For either movement process, $K$ decreased exponentially as $Z$ increased. The rate of decrease was much greater in the BW case since each animal tended to move across the territory in a much shorter timescale than the NNRW case. This caused each point on the territory border to be rescented at shorter time intervals so that the borders moved less. As an indicator of how these timescales differ, if the territories were immobile and square, the territory width would be $1 /(\rho)^{1 / 2}$ and the area would be $1 / \rho$. Therefore, the respective timescales would be proportional to $1 /(\rho)^{1 / 2}$ for the BW case, the time it takes for a ballistic walker to move a distance of $1 /(\rho)^{1 / 2}$, compared to $1 / \rho$ for NNRW, the first passage time for such a walker to traverse a distance of $1 /(\rho)^{1 / 2}$ (see, e.g., Redner 2007, section 2.4).

\section{Territorial Dynamics of a Red Fox Population before and after an Outbreak of Sarcoptic Mange}

During the 1994-1996 mange epizootic, the red fox population density declined rapidly, causing the foxes to both extend their home ranges and move faster (Baker et al. 2000). To investigate further the effect of population decline on the animals' movements and interactions, we fitted the data to the model of animal movement in a territory of fluctuating size and position (Giuggioli et al. 
Table 1: Glossary of terms and best-fit values

\begin{tabular}{|c|c|c|c|c|}
\hline Symbol & Model & Explanation & Premange & Postmange \\
\hline$K$ & A & Territory border diffusion constant $\left(\mathrm{m}^{2} / \mathrm{min}\right)$ & $3.97 \pm .09$ & $32.8 \pm .6$ \\
\hline $\mathcal{T}$ & A & Correlation time (min; see "Methods") & $14.9 \pm .5$ & $14.8 \pm .4$ \\
\hline$L$ & A & Average territory width $(\mathrm{m})$ & $435 \pm 37$ & $926 \pm 44$ \\
\hline$\gamma$ & A & $\begin{array}{l}\text { Territory renormalization rate }\left(\mathrm{min}^{-1}\right) \text { : rate at which a } \\
\text { territory tends to return to an average area of } 1 / L^{2}\end{array}$ & $108 \pm 2$ & $140 \pm 2$ \\
\hline$v$ & A, S & Average animal velocity $\left(\mathrm{m} \mathrm{min}^{-1}\right)$ & $7.39 \pm .16$ & $18.9 \pm .3$ \\
\hline$\rho$ & A, S & Population density $\left(\mathrm{km}^{-2}\right)$ & $5.29 \pm 1.02$ & $1.17 \pm .12$ \\
\hline$T_{*}$ & A, S & Time window over which home range is measured (days) & 84.0 & 84.0 \\
\hline$T_{\mathrm{AS}}$ & S & Active scent time (days) & $5.07 \pm .55$ & $3.37 \pm .16$ \\
\hline$T_{\text {at }}$ & S & Territorial acquisition time (days) & $\ldots$ & $4.89 \pm .16$ \\
\hline$a$ & S & Lattice site separation (length) & $\ldots$ & $\ldots$ \\
\hline$\tau$ & $S$ & $\tau=a / v($ time $)$ & $\ldots$ & $\ldots$ \\
\hline$T_{\mathrm{C}}$ & S & $T_{\mathrm{C}}=1 /\left(v^{2} \tau \rho\right)($ time $)$ & $\ldots$ & $\ldots$ \\
\hline$Z$ & $S$ & $Z=T_{\mathrm{AS}} / T_{\mathrm{C}}$ (dimensionless) & $10.5 \pm .2$ & $10.0 \pm .2$ \\
\hline
\end{tabular}

Note: Glossary of parameters for both the analytic model of animal movement inside slowly fluctuating territory borders and the simulation model of territorial random walkers, together with their values for the fox data premange and postmange, if applicable. Error range is 1 standard deviation using the bootstrap algorithm (see main text for details). The second column details whether the parameter is used in the analytic model (A) or simulation model (S).

2012). Table 1 details the parameters $\theta=(v, K, \mathcal{T}, \gamma, L)$ that gave the best fits of the premange and postmange data sets to the probability distribution $P(x, y, t \mid \theta)$ for an animal moving within its territory. Figure 3 shows all the premange fox positions, superimposed on the utilization distribution of the model, which measures the expected distribution of animal fixes across a season (see "Analytic Expression of the Probability Distribution for an Animal in a Slowly Moving Territory"). Video 1 shows the evolution of both the fox positions and the model's probability distribution over time.

As well as the foxes having a much larger average velocity after the mange outbreak, the value of $K$ increased more than eightfold, meaning that territory borders moved much more rapidly after the population density declined. As the foxes died out, neighboring foxes took over the newly vacated areas, causing the borders to move and the territories to enlarge, as evidenced by an increase in average territory size from $L^{2}=0.189 \mathrm{~km}^{2}$ premange to $L^{2}=0.857 \mathrm{~km}^{2}$ postmange. In table 1 , we present values of the territory width $L$, which is the square root of its area. The increase in $\gamma$ after the mange outbreak suggests that territories were pushed toward an average size faster when the population density was less, in keeping with the idea that territories were more "elastic" during the postmange period.

While the Bristol foxes increased $v$ as the population density dropped, surprisingly, they did not increase $\mathcal{T}$. That is, the turning angle distribution had similar statistics both before and after the mange outbreak. However, due to their increased velocity, the distance for which they would persist in roughly the same direction, $v \mathcal{T}$, was 2.5 times higher postmange. In other words, the mean step length between turns was higher. The lack of change in turning angle distribution may indicate an inbuilt species- or habitat-specific search strategy.

The expected percentage of home range overlap, HRO, was measured over a time window of $T_{*}=84.0$ days, which was the maximum time between the first and the last location fix for any of the seasons during which data were gathered. It was calculated using equation (1) to be $\mathrm{HRO}=24.7 \% \pm 3.3 \%$ premange and $\mathrm{HRO}=$ $30.6 \% \pm 2.5 \%$ postmange (error bars are $1 \mathrm{SD}$ ). This apparent increase in overlap is not statistically significant $(P=.07)$. However, given that there were only four individuals tracked in the postmange period and that the data during that period were dominated by two of these four (see table A1), this increase is not negligible. The failure of this test to reject the null hypothesis that there is no increase in overlap may therefore be subject to a Type II error (see, e.g., Wasserman 2004), so this result should be considered tentative. Previous studies using this data set (Baker et al. 2000) used MCP techniques to measure the overlaps directly. Though MCP techniques have in recent years been shown to be less than ideal (Laver and Kelly 2008), the study by Baker et al. (2000) also showed an apparent small increase in home range overlap that was not statistically significant.

Were $T_{\mathrm{AS}}, v$, and $\mathcal{T}$ to have remained constant as the population density decreased, there would have been a dependency of the home range overlap on the population density of the type shown in the inset of figure 2 (solid curve). In such a case, unless the density is very low, the percentage of overlap decreases as the population density increases. However, for extremely low densities, neighboring 


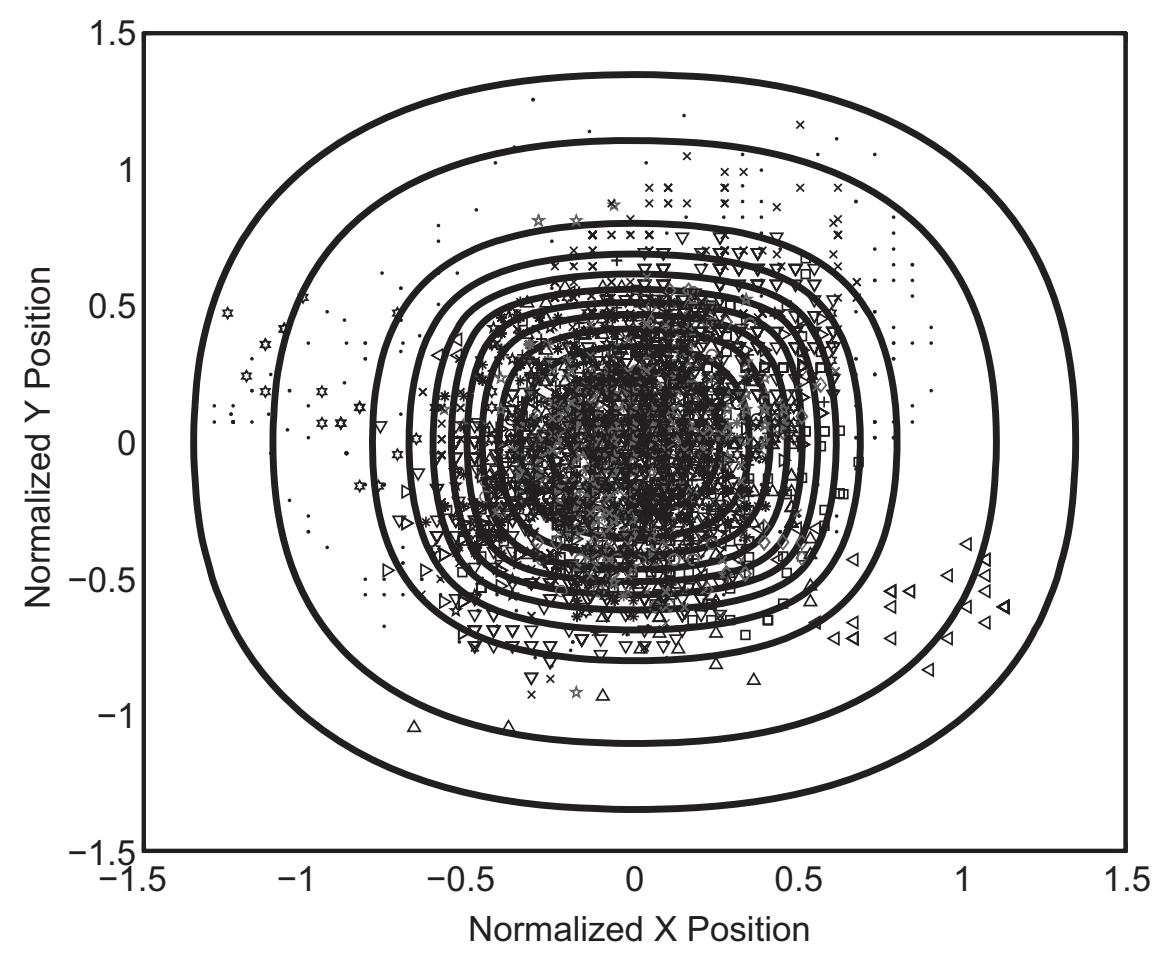

Figure 3: The utilization distribution of the analytic model of animal movement inside a slowly moving territory, with location fixes of Bristol foxes before the 1994 outbreak of sarcoptic mange $(N=8,693)$, and parameter values fitted to the premange data. The fixes have been normalized so that the center of each seasonal home range is at position $(0,0)$ and the distances from the center have been divided by $L=435 \mathrm{~m}$, the average territory width. Different symbols denote different foxes. The contours are placed at deciles $(1 / 10,2 / 10,3 / 10$, etc.) of the distribution height, except for the outer two, which are placed at $1 / 100$ and $1 / 1,000$ of the height. See video 1 to view the evolution of the probability distribution over time.

animals would be so far apart that they are unlikely to encounter one another's territorial borders, meaning the ranges would overlap little. This trend in home range overlap implies that the home range size HRS $=L^{2}\{1+$ $\left.\left[K T_{*} \rho / \ln \left(T_{*} / \tau\right)\right]^{1 / 2}\right\}^{2}$ is proportional to $\rho^{-\alpha}$ where $\alpha>1$, assuming fixed $T_{\mathrm{AS}}, v$, and $\mathcal{T}$. The home range size is estimated by taking the square of the home range width $L\{1+$ $\left.\left[K T_{*} \rho / \ln \left(T_{*} / \tau\right)\right]^{1 / 2}\right\}$. Using the premange values of these parameters, we fitted a straight line to the plot of $\log$ (HRS) against $\log (\rho)$ (using linear least squares) to find that $\alpha \approx$ 1.74 .

\section{Inferring Active Scent Time from Location Data}

The value of $T_{\mathrm{AS}}$ for the fox population was found by using the best-fit values of $K$ from the analytic model together with the NNRW trend curve from the simulation output (fig. 2). Since animals that move with a correlation time $\mathcal{T}$ appear to be uncorrelated random walkers when sampled at a temporal resolution lower than or equal to $\mathcal{T}$ (i.e., the time between fixes is greater than $\mathcal{T}$ ), the time it takes to move between adjacent lattice sites was set equal to the correlation time, that is, $\tau=\mathcal{T}$.

Though we analyzed both ballistic and random walks in our simulation model, the foxes we studied tended to make several turns between one visit to the border and the next. This is evident from the correlation time $\mathcal{T}$ of just under $15 \mathrm{~min}$ (table 1), which is insufficient time for the foxes to traverse their territories at average speed (e.g., $7.39 \mathrm{~m} \mathrm{~min}^{-1}$ premange, with a territory width of $435 \mathrm{~m}$ ). Therefore, we restrict our data analysis to using the random walk version of the simulation model.

For the premange data, the dimensionless value $K / v^{2} \mathcal{T}$ was $4.89 \pm 0.54 \times 10^{-3} \quad(\mathrm{SD})$, whereas postmange $K / v^{2} \mathcal{T}=6.19 \pm 0.56 \times 10^{-3}(\mathrm{SD})$. The best-fit curve from the NNRW simulation output, $\log _{10}\left(K / v^{2} \mathcal{T}\right)=$ $0.085-0.247 Z$, gives $Z=10.5 \pm 0.2(\mathrm{SD})$ premange and $Z=10.0 \pm 0.2(\mathrm{SD})$ postmange. To link the simulation parameters to the analytic model, the population density was assumed to be $\rho=1 / L^{2}$, and was $5.29 \pm 1.02 \mathrm{~km}^{-2}$ (SD) fox territories premange and $\rho=1.16 \pm 0.12 \mathrm{~km}^{-2}$ (SD) postmange. Therefore, $T_{\mathrm{AS}}=Z /\left(v^{2} \mathcal{T} \rho\right)$ was $5.07 \pm$ 


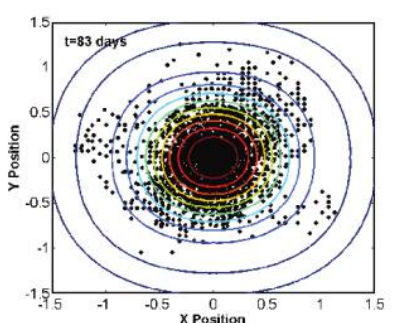

Video 1: Video 1, available online, shows how the probability distribution $P(x, y, t \mid \theta)$ evolves through time. The contours on the video show $P(x, y, t \mid \Theta)$ for the values of $\Theta$ that best fit the premange data (see main text). The dots on the video show cumulative locations of foxes through time from the premange data set, normalized so that the centers of their home ranges are all at $(0,0)$ and the distances from the center are divided by $L=435 \mathrm{~m}$.

0.55 days $(\mathrm{SD})$ premange and $3.37 \pm 0.16$ days $(\mathrm{SD})$ postmange, a statistically significant decrease $(P=.002)$.

We also used the active scent time to infer how long it took for a neighbor to seize a territory once it had been vacated, the so-called territory acquisition time $T_{\mathrm{at}}$. By running NNRW simulations whereby one fox is removed part way through, we measured the time it took for other foxes to seize $90 \%$ of the dead fox's territory (see video 2). Except when the population density was very low, the dimensionless value $\left(T_{\mathrm{at}}-T_{\mathrm{AS}}\right) \rho v^{2} \tau$ tended to range between about 3.5 and 5 (see "Calculating Territorial Acquisition Time," available online). For the postmange case of $Z=10.0$, the value of $\left(T_{\mathrm{at}}-T_{\mathrm{As}}\right) \rho v^{2} \tau$ was found to be 4.50 , by averaging over various values of $\rho, v, \tau$, and $T_{\mathrm{AS}}$ such that $Z=10.0$. This implies that the territory acquisition time $T_{\text {at }}$ was approximately 5 days.

\section{Discussion}

By applying recently developed agent-based models of territory formation to a natural experiment in a red fox population, we have quantified the changes in both territorial patterns and individual behavior elicited by a rapid population decline in a paradigmatic territorial species. Our modeling framework enabled us to relate elasticity in territory borders directly to the individual-level movement and interaction mechanisms, allowing information about territorial dynamics to be inferred from animal movement data. We have constructed a program for making these inferences, when interactions are scent mediated, by fitting a time-evolving probability distribution to spatiotemporal location data, and we have applied this to data on red fox movements. This program gives a way of quantifying the mechanisms underpinning the elastic disc hypothesis (Huxley 1934).

As the 1994-1996 epizootic of sarcoptic mange deci- mated the Bristol fox population, territorial patterns changed rapidly, affecting both the foxes' movement and interaction processes. Fluctuations of territory borders became more pronounced, evidenced by an eightfold increase in the territory diffusion constant $K$. The animals increased their average speed of travel by around 2.5 times. We also observed a decrease in the active scent time. These behavioral changes enabled the foxes to increase their territory size to maintain contiguous borders and thereby exclude potential invaders (Baker et al. 2000), while ensuring that the percentage of home range overlap remained roughly constant.

The decrease in active scent time from 5 days to just over 3 days meant that foxes waited for a shorter time before attempting to acquire territorial area that they believed had been vacated. As well as scent, foxes use vocal cues to inform neighbors of their presence (Newton-Fisher et al. 1993). The absence of these additional cues after the death of a fox could suggest to neighbors that the territory had been vacated, and so they may be more willing to venture into areas that contain scent that is only 3 or 4 days old. There may also have been a reduction in the number of scent marks left by foxes in the terminal stages of sarcoptic mange. While scent marks contain information about health and status (Arnold 2009), a territory was not invaded until after the neighbors had died (Baker et al. 2000), so it appears that health did not influence active scent time in foxes.

Overlapping home ranges emerge in the model as a direct outcome of fluctuating territory borders, without requiring animals to wander into neighboring territories. While the home range is a measurement of the utilization distribution across a period of time such as a day, month, or season, the territory is the area being defended, for

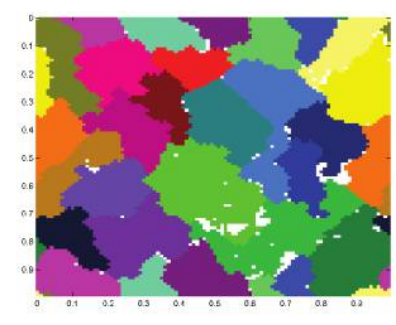

Video 2: Video 2, available online, shows the dynamics of territorial acquisition. It shows 25 territorial random walkers on a $100 \times$ 100-square lattice with periodic boundary conditions. Each territory is denoted by a different color. The white squares are interstitial regions, where there is no active scent. Partway through the video, the simulated animal with the cyan territory is removed, shown by the color of the territory turning black. As the scent of this animal becomes inactive, the black squares turn white, allowing the other animals to move in and acquire the territory. 
example, by fresh scent marks, at any point in time. As the locations of an animal are measured over time, its territory varies in both position and size. These fluctuations may be difficult to detect in ecological studies, since by the time sufficient location fixes have been obtained to measure territory size, using either KDE (Worton 1989) or other techniques (as reviewed in Fieberg and Börger 2012), the borders may have changed. Consequently, if the fluctuating territories are contiguous, the utilization distributions measured over a period of time will overlap (Giuggioli et al. 2011a). So overlap may simply be an artifact of the timescale for the data collection, due to home range size increasing over time (Börger et al. 2006), and not an implicit biological phenomenon, although there may be circumstances where it is related to competition for resources (Buchmann et al. 2010) or territorial intrusion (Burt 1943).

Though we used random walks to model fox movement in Bristol's resource-rich environment, movement processes in a resource-poor environment probably exhibit a certain amount of heterogeneity, dependent on the spatial distribution of food resources (Tremblay et al. 2007). It is evident from the comparison of two simple animal movement processes, ballistic motion and diffusive random walks, that the nature of the animal movement greatly affects the territory border dynamics (fig. 2). Recent studies by Benhamou (2011) and Benhamou and Riotte-Lambert (2012) use movement-based kernel density estimations and Brownian bridge techniques to determine the heterogeneous movement processes that arise from animals searching for food within their home ranges. In the future, more complicated movement processes, caused either by resource distribution or other ecological phenomena (e.g., Morales et al. 2004; Reynolds 2010), could be built into our modeling framework to quantify territorial dynamics in populations where the movement cannot be realistically modeled by a simple random walk.

As well as providing insights into behavioral ecology, these results have important consequences for modeling epizootic spread, particularly when the disease is terminal or restricts movement and hence scent-marking behavior. Rather than assuming constant territory or home range sizes (e.g., Smith and Harris 1991; Smith and Wilkinson 2002; Kenkre et al. 2007; Salkeld et al. 2010), our results demonstrate that the rapid decline of population density elicited by terminal disease spread causes large changes in the territorial dynamics. This in turn affects the behavior of the animals and the interanimal contact rates. Our approach provides the necessary basis to enable future epidemiological studies to take such behavioral and territorial fluctuations into account, allowing for improved predictions of disease spread.

\section{Acknowledgments}

This work was partially supported by Engineering and Physical Sciences Research Council grants EP/E501214/1 (J.R.P.) and EP/I013717/1 (L.G.) and by the Dulverton Trust (S.H). We thank the editors and two anonymous reviewers, whose comments helped improve the article.

\section{Literature Cited}

Adams, E. S. 2001. Approaches to the study of territory size and shape. Annual Review of Ecology, Evolution, and Systematics 32:277-303. Arnold, J. 2009. Olfactory communication in red foxes (Vulpes vulpes). $\mathrm{PhD}$ thesis, University of Bristol.

Baker, P., S. M. Funk, S. Harris, and P. C. L. White. 2000. Flexible spatial organization of urban foxes, Vulpes vulpes, before and during an outbreak of sarcoptic mange. Animal Behaviour 59:127-146.

Beier, P. 1993. Determining minimum habitat areas and habitat corridors for cougars. Conservation Biology 7:94-108.

Benhamou, S. 2011. Dynamic approach to space and habitat use based on biased random bridges. PLoS ONE 6:e14592.

Benhamou, S., and L. Riotte-Lambert. 2012. Beyond the utilization distribution: identifying home range areas that are intensively exploited or repeatedly visited. Ecological Modelling 227:112-116.

Börger, L., B. Dalziel, and J. M. Fryxell. 2008. Are there general mechanisms of animal home range behavior? a review and prospects for future research. Ecology Letters 11:637-650.

Börger, L., N. Franconi, F. Ferretti, F. Meschi, G. De Michele, A. Gantz, and T. Coulson. 2006. An integrated approach to identify spatiotemporal and individual-level determinants of animal home range size. American Naturalist 168:471-485.

Briscoe, B. K., M. A. Lewis, and S. E. Parrish. 2002. Home range formation in wolves due to scent marking. Bulletin of Mathematical Biology 64:261-284.

Buchmann, C. M., F. M. Schurr, R. Nathan, and F. Jeltsch. 2010. An allometric model of home range formation explains the structuring of animal communities exploiting heterogeneous resources. Oikos 120:106-118.

Burt, W. H. 1943. Territoriality and home range concepts as applied to mammals. Journal of Mammalogy 24:346-352.

Condamin, S., O. Bénichou, and M. Moreau. 2007. Random walks and Brownian motion: a method of computation for first-passage times and related quantities in confined geometries. Physical Review E 75:021111.

Durrett, R., and S. Levin. 1994. The importance of being discrete (and spatial). Theoretical Population Biology 46:363-394.

Ezaki, Y. 1995. Establishment and maintenance of the breeding territory in the polygynous great reed warbler. Ecological Research 10:359-368.

Fieberg, J., and L. Börger. 2012. Could you please phrase "home range" as a question? Journal of Mammalogy 93:890-902.

Giuggioli, L., J. R. Potts, and S. Harris. 2011a. Animal interactions and the emergence of territoriality. PLoS Computational Biology 7:1002008.

- 2011b. Brownian walkers within subdiffusing territorial boundaries. Physical Review E 83:061138.

2012. Predicting oscillatory dynamics in the movement of territorial animals. Iournal of the Roval Societv Interface 9:15291543. 
Grant, J. W. A., and D. L. Kramer. 1990. Territory size as a predictor of the upper limit to population density of juvenile salmonids in streams. Canadian Journal of Fisheries and Aquatic Sciences 47: $1724-1737$.

Harris, S., W. J. Cresswell, P. G. Forde, W. J. Trewhella, T. Woollard, and S. Wray. 1990. Home-range analysis using radio-tracking data: a review of problems and techniques particularly as applied to the study of mammals. Mammal Review 20:97-123.

Hutchings, M. R., K. M. Service, and S. Harris. 2001. Defecation and urination patterns of badgers Meles meles at low density in southwest England. Acta Theriologica 46:87-96.

Huxley, J. S. 1934. A natural experiment on the territorial instinct. British Birds 27:270-277.

Jetz, W., C. Carbone, J. Fulford, and J. H. Brown. 2004. The scaling of animal space use. Science 306:266-268.

Kenkre, V. M., L. Giuggioli, G. Abramson, and G. Camelo-Neto. 2007. Theory of hantavirus infection spread incorporating localized adult and itinerant juvenile mice. European Phvsical Journal B 55:461-470.

Kenward, R. E., R. T. Clarke, K. H. Hodder, and S. S. Walls. 2001. Density and linkage estimators of home range: nearest-neighbor clustering defines multinuclear cores. Ecology 82:1905-1920.

Kruuk, H., and T. Parish. 1982. Factors affecting population density, group size and territory size of the European badger, Meles meles. Lournal of Zoology (London) 196:31-39.

Lagarias, J. C., J. A. Reeds, M. H. Wright, and P. E. Wright. 1998. Convergence properties of the Nelder-Mead simplex method in low dimensions. SIAM Journal of Optimization 9:112-147.

Landim, C. 1992. Occupation time large deviations for the symmetric simple exclusion process. Annals of Probability 20:206-231.

Laver, P. N., and M. J. Kelly. 2008. A critical review of home range studies. Journal of Wildlife Management 72:290-298.

Lewis, M. A., and J. D. Murray. 1993. Modelling territoriality and wolf-deer interactions. Nature 366:738-740.

Liggett, T. M. 1985. Interacting particle systems. Springer, New York.

Macdonald, D. W. 1980. Patterns of scent marking with urine and faeces amongst carnivore communities. Symposia of the Zoological Society of London 45:107-139.

McCarthy, K. P., and S. Destefano. 2011. Effects of spatial disturbance on common loon nest site selection and territory success. Journal of Wildlife Management 75:289-296.

McKane, A. J., and T. J. Newman. 2004. Stochastic models in population biology and their deterministic analogs. Physical Review E 70:041902.

Moorcroft, P. R., and A. Barnett. 2008. Mechanistic home range models and resource selection analysis: a reconciliation and unification. Ecology 89:1112-1119.

Moorcroft, P. R., and M. A. Lewis. 2006. Mechanistic home range analysis. Princeton University Press, Princeton, NJ.

Moorcroft, P. R., M. A. Lewis, and R. L. Crabtree. 2006. Mechanistic home range models capture spatial patterns and dynamics of coyote territories in Yellowstone. Proceedings of the Roval Society B: Biological Sciences 273:1651-1659.

Morales, J. M., D. T. Haydon, J. Friar, K. E. Holsinger, and J. M. Fryxell. 2004. Extracting more out of relocation data: building movement models as mixtures of random walks. Ecology 85:2436-2445.

Newton-Fisher, N., S. Harris, P. White, and G. Jones. 1993. Structure and function of red fox Vulpes vulpes vocalisations. Bioacoustics 5: $1-31$.
Okubo, A., and S. A. Levin. 2002. Diffusion and ecological problems: modern perspectives. 2nd ed. Springer, New York.

Potts, J. R., S. Harris, and L. Giuggioli. 2011. An anti-symmetric exclusion process for two particles on an infinite 1-D lattice Journal of Physics A 44:485003.

- 2012. Territorial dynamics and stable home range formation for central place foragers PLoS ONE 7:e34033.

- 2013. Data from: Quantifying behavioral changes in territorial animals caused by sudden population declines. American Naturalist, Dryad Digital Repository, http://dx.doi.org /10.5061/dryad.5dn48.

Redner, S. 2007. A guide to first-passage processes. Cambridge University Press, Cambridge.

Reynolds, A. M. 2010. Animals that randomly reorientate at cues left by correlated random walkers do the Lévy walk. American Naturalist 175:607-613.

Salkeld, D. J., M. Salathé, P. Stapp, and J. H. Jones. 2010. Plague outbreaks in prairie dog populations explained by percolation thresholds of alternate host abundance. Proceedings of the National Academy of Sciences of the USA 107:14247-14250.

Saunders, G., P. C. L. White, S. Harris, and J. M. V. Rayner. 1993. Urban foxes (Vulpes vulpes): food acquisition, time and energy budgeting of a generalized predator. Symposia of the Zoological Society of London 65:215-234.

Schradin, C., G. Schmohl, R. G. Rödel, I. Schoepf, S. M. Treffler, J. Brenner, M. Bleeker, M. Schubert., B. König, and N. Pillay. 2010. Female home range size is regulated by resource distribution and intraspecific competition: a long-term field study. Animal Behaviour 79:195-203.

Smith, G. C., and S. Harris. 1991. Rabies in urban foxes (Vulpes vulpes) in Britain: the use of a spatial stochastic simulation model to examine the pattern of spread and evaluate the efficacy of different control regimes. Philosophical Transactions of the Royal Society B: Biological Sciences 334:459-479.

Smith, G. C., D. Wilkinson. 2002. Modelling disease spread in a novel host: rabies in the European badger Meles meles. Iournal of Applied Ecology 39:865-874.

Stamps, J. A., and V. V. Krishnan. 1998. Territory acquisition in lizards. IV. Obtaining high status and exclusive home ranges. Animal Behaviour 54:461-472.

Stutchbury, B. J. 1991. Floater behavior and territory acquisition in male purple martins. Animal Behaviour 42:435-443.

Tremblay, Y., A. J. Roberts, and D. P. Costa. 2007. Fractal landscape method: an alternative approach to measuring area-restricted searching behavior. Journal of Experimental Biology 210:935-945.

Van Moorter, B., D. Visscher, S. Benhamou, L. Börger, M. S. Boyce, and J. M. Gaillard. 2009. Memory keeps you at home: a mechanistic model for home range emergence. Oikos 118:641-652.

Viswanathan, G. M., E. P. Raposo, F. Bartumeus, J. Catalan, and M. G. E. da Luz. 2005. Necessary criterion for distinguishing true superdiffusion from correlated random walk processes. Physical Review E 72:011111.

Wasserman, L 2004. All of statistics: a concise course in statistical inference. 2nd ed. Springer, New York.

Worton, B. J. 1989. Kernel methods for estimating the utilization distribution in home-range studies. Ecology 70:164-168.

Associate Editor: Peter Nonacs Editor: Judith L. Bronstein 